\title{
Étude comparée de la croissance osseuse diaphysaire du tibia entre 3 et 12 semaines dans 2 souches de dindes: aspects histologiques et histomorphométriques
}

\author{
M Wyers, Y Cherel, G Plassiart, É Chabeauti, V Mariau, \\ B Fernandez, C Guéreaud, L Guigand
}

\begin{abstract}
École nationale vétérinaire, laboratoire d'anatomie pathologique, Unité associée INRA : "Système locomoteur de la dinde», CP 3013, F 44087 Nantes cedex 03, France
\end{abstract}

(Reçu le 7 décembre 1992 ; accepté le 17 août 1993)

\begin{abstract}
Résumé - La croissance en épaisseur de la diaphyse tibiale a été comparée chez les mâles de 2 souches de dindes, âgés de 3 à 12 semaines, une souche légère de type fermier et une souche lourde de sélection. La croissance en épaisseur est beaucoup plus rapide et plus intense chez les dindes de souche lourde, chez qui la forme ovoïde de la diaphyse révèle l'action de fortes contraintes mécaniques. En revanche le pourcentage d'os cortical élaboré demeure le même, dans les 2 souches, dès la $3^{e}$ semaine. Durant toute la période étudiée, la souche légère montre des variations plus fréquentes de la vitesse d'apposition osseuse ainsi que des phénomènes de remodelage endostéal plus précoces et plus étendus. La comparaison des paramètres de croissance diaphysaire chez les animaux des 2 souches de poids comparable montre que la plupart des paramètres sont, à poids égal, supérieurs chez les dindons de souche lourde.
\end{abstract}

tibio-tarse / diaphyse / croissance / dindon / histologie / histomorphométrie

Summary - Comparative histological and histomorphometrical studies of the tibial diaphyseal growth in 2 turkey strains between the 3rd and the 12th weeks of age. The comparative cortical growth of the tibiotarsus was studied in 2 turkey strains, one light, one heavy, between 3 and 12 weeks of age, using histological and histomorphometrical methods. The growth in thickness started earlier and was much faster and larger in the heavier turkey strain. The early ovoid shape of the cortex of the heavier strain and the difference between the thickness of the narrow and the thick cortical faces seemed to be related to the action of strong mechanical strains. Nevertheless the diaphyseal cortical ratio remained not significantly different in the 2 strains from the age of 3 weeks. The light strain exhibited during all the studied periods indicated a more variable mineral apposition rate and earlier and more extensive bone remodelling phenomena. Most of the growth in thickness parameters were significantly higher in the heavier strain when turkeys of the 2 strains were compared at an equivalent weight. 


\section{INTRODUCTION}

Les troubles locomoteurs représentent chez la dinde en croissance un problème fréquent et grave (Sanger et al, 1974; Wise, 1975 ; Riddell, 1975, 1981). Leur étiologie et leur pathogénie demeurent actuellement encore mal comprises, en revanche leur expression clinique commence à être mieux connue (Cherel et al, 1991a).

En quelques dizaines d'années, la sélection génétique a permis d'obtenir des souches de dinde extrêmement différentes tant sur le plan du poids que de la vitesse de croissance, permettant de distinguer notamment des souches dites "fermières", d'un poids moyen d'environ $7 \mathrm{~kg}$ chez le mâle adulte de $32-33$ semaines et des souches «lourdes", utilisées à des fins de sélection où le poids des mâles adultes atteint couramment $30 \mathrm{~kg}$. Chez ces derniers, 20 à $30 \%$ des animaux sont éliminés au cours de leur période de croissance du fait de l'apparition de troubles locomoteurs (Sté Bétina, communication personnelle).

Les troubles locomoteurs existent également fréquemment chez les dindons mâles de souches commerciales "médium alourdies" où ils s'expriment habituellement vers l'âge de $10-12$ semaines (Cherel et al, 1991a).

Les différences morphologiques et cliniques constatées entre les souches de dinde ont conduit à entreprendre l'étude comparée de la croissance de l'appareil locomoteur d'une souche fermière et d'une souche lourde de sélection afin de rechercher des facteurs susceptibles d'expliquer ou de favoriser l'apparition des troubles locomoteurs dans les souches les plus lourdes (Abourachid, 1990).

Une étude histologique générale de la croissance du tibia (Leblanc et al, 1986) a montré des différences à certains âges entre les 2 souches aussi bien de la croissance en longueur que de la croissance en épaisseur de l'os. Une étude plus récente (Wyers et al, 1993) a confirmé que la croissance diaphysaire était plus précoce et plus rapide chez les jeunes animaux de la souche lourde, bien que le pourcentage de surface corticale par rapport à la surface diaphysaire totale demeure comparable dans les 2 souches.

La présente étude complète et précise les 2 observations précédentes. Elle envisage dans les 2 mêmes souches de dinde les caractères histologiques qualitatifs et quantitatifs de la croissance diaphysaire du tibia entre 3 et 12 semaines.

\section{MATÉRIEL ET MÉTHODES}

\section{Animaux}

Les animaux sont des dindons mâles, cliniquement normaux, appartenant aux 2 souches utilisées au cours des études précédentes (Leblanc et al, 1986; Wyers et al, 1993) et fournis par la société Bétina Sélection (56250 Elven) :

- une souche légère de type souche «fermière» commercialisée sous l'appellation de «dinde de Noël» ;

- une souche "lourde" utilisée en sélection.

Les animaux ont été élevés dans des conditions standard et ont reçu ad libitum un aliment conçu et fabriqué par la société Guyomarc'h (Vannes, Morbihan). La composition de l'aliment a été publiée précédemment (Leblanc et al, 1986)

Huit lots de 15 animaux âgés respectivement de $3,5,6,7,8,9,10$, et 12 semaines ont été choisis dans les 2 souches.

Afin d'étudier par observation en fluorescence, différents paramètres de la croissance osseuse, chaque dindon a reçu, par voie intrapéritonéale, 2 injections d'un fluorochrome : I'oxytétracycline (Terramycine ND Pfizer) à une semaine d'intervalle à la dose de $30 \mathrm{mg}$ par $\mathrm{kg}$ de poids vif. La $2^{\mathrm{e}}$ injection a été pratiquée $2 \mathrm{j}$ avant l'euthanasie. 
Les dindons, acheminés vivants au laboratoire, ont été sacrifiés par une injection intraveineuse d'un produit d'euthanasie commercialisé sous le nom de T 61 ND (UVECO), saignés puis pesés.

\section{Réalisation et traitement des prélèvements}

Le tibio-tarse gauche a été débarrassé de ses masses musculo-tendineuses et sa longueur a été mesurée à l'aide d'un double décimètre. La face craniale de l'os a été identifiée par un trait de marquage indélébile afin de conserver l'orientation de la diaphyse au cours des manipulations ultérieures puis une section transversale a été réalisée au centre du rayon osseux.

Une section longitudinale de l'épiphyse proximale du tibio-tarse a également été pratiquée pour mettre en évidence la présence éventuelle de lésions de dyschondroplasie tibiale.

À partir de la section médiane transversale du rayon osseux, un fragment diaphysaire d'environ $1 \mathrm{~cm}$ de haut a été prélevé sur la partie supérieure de la diaphyse, fixé dans du formol tamponné, déshydraté et inclus en résine plastique (Méthyl-Métacrylate) selon la méthode de Schenck et al (1984) adaptée au laboratoire à l'étude du tissu osseux de la dinde (Chabeauti, 1988 ; Mariau, 1988).

Le bloc a été ensuite sectionné à l'aide d'une meule diamantée (Accutom). Les lames ainsi obtenues, d'une épaisseur initiale de $200 \mu \mathrm{m}$, ont été ensuite collées sur une lame de plexiglas et polies à l'aide de pâtes diamantées (Polisseuse DAP 7 STRUERS) jusqu'à une épaisseur finale de $100 \mu \mathrm{m}$ pour obtenir une lame dite "mince" observable au microscope (Hutzchnenreuter et Brummer, 1984).

Le marquage indélébile préalable de la face craniale de la diaphyse permet de repérer celleci sur les lames minces ainsi réalisées.

Une seule lame mince a été réalisée et examinée à partir du bloc inclus en résine plastique, c'est-à-dire une seule lame par animal. Le nombre final de lames exploitables varie selon les lots (tableau I) ; il est très faible dans la souche lourde à 10 sem en raison d'un nombre élevé d'animaux porteurs de lésions de dyschondroplasie tibiale. Nous avons, en effet, éliminé de notre étude les animaux dyschondro-
Tableau I. Nombre de lames exploitables et effectif des lots.

$$
\begin{array}{llllllll}
\multicolumn{4}{c}{\text { Âges (en sem) }} \\
3 & 5 & 6 & 7 & 8 & 9 & 10 & 12
\end{array}
$$

Souche fermière

$\begin{array}{llllllll}9 & 12 & 12 & 9 & 8 & 10 & 13 & 7\end{array}$

Souche ultra$\begin{array}{llllllll}10 & 10 & 12 & 11 & 10 & 9 & 3 & 9\end{array}$ lourde

Une seule lame du tibio-tarse gauche a été traitée par animal.

plasiques en raison des répercussions probables de cette lésion sur la forme et la structure de la diaphyse (Chérel et al, 1991b).

Les préparations ont été examinées, soit sans coloration à l'aide d'un microscope à fluorescence (Leitz Orthoplan obj $\times 25$ oculaire $\times$ $6,5)$, soit après coloration par le trichrome de Goldner (Villanueva et Mehe, 1977).

Afin d'obtenir des images de la totalité de la surface de section diaphysaire à tous les âges étudiés, des photographies de lames minces non colorées ont été réalisées sur fond noir à la loupe binoculaire (Leitz). Chaque préparation était accompagnée d'une échelle graphique en papier millimétré photographiée et développée dans des conditions identiques à celles du cliché de la diaphyse.

\section{Étude qualitative}

Un cadran de papier transparent divisé en 8 sections (fig 1) a été utilisé pour localiser les différents territoires diaphysaires sur les photographies de section transversale. Le centre du cadran étant placé au centre approximatif de la diaphyse et la marque identifiant la zone craniale dans la portion $n^{\circ} 1$. II a été possible de déterminer ainsi dans quelle portion se localisent la section de l'artère nourricière, la zone la plus mince ou la zone la plus épaisse de la corticale diaphysaire.

La structure osseuse de la corticale a été étudiée en fluorescence ou après coloration par le trichrome de Goldner. 


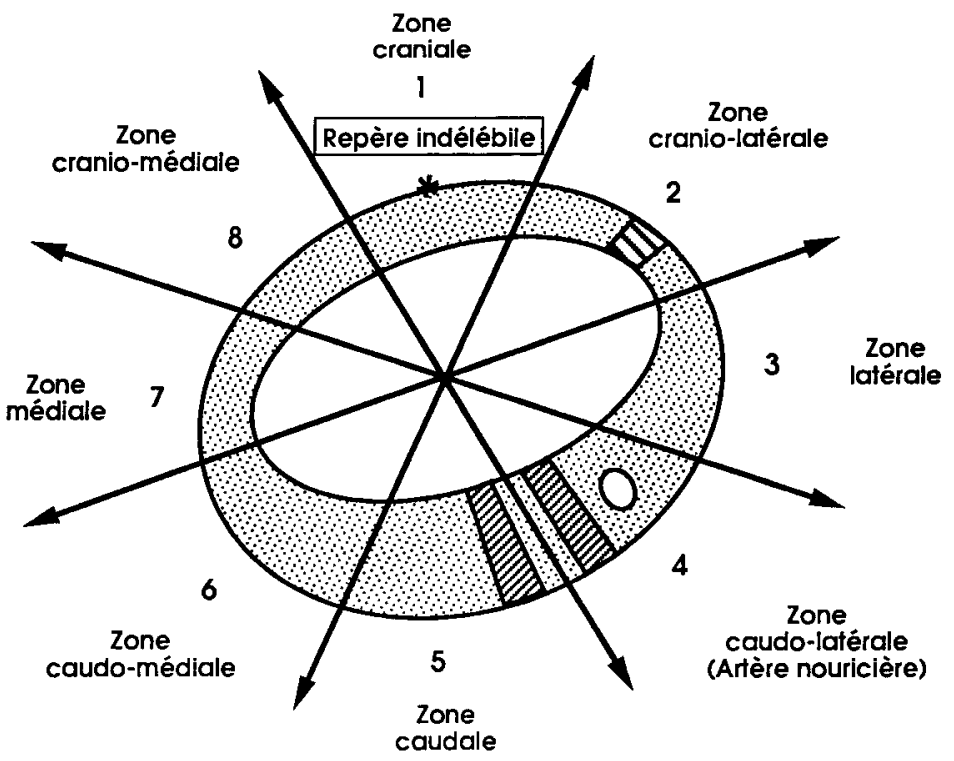

Fig 1. Méthode de la localisation des différents territoires diaphysaires. $\bar{Z}$ Zone mince (ZMd); Zone épaisse (ZEd).

La disposition vasculaire du tissu cortical fibro-lamellaire a été distinguée selon la classification proposée par De Ricqles $(1975,1977)$.

\section{Étude quantitative : définition des paramètres histomorphométriques}

Plusieurs paramètres histomorphométriques destinés à quantifier la croissance en épaisseur de la diaphyse ont été appréciés à l'aide d'un analyseur d'images semi-automatique ASM (Leitz).

Les paramètres suivants ont été mesurés ou calculés à partir des photographies de diaphyse:

- le périmètre diaphysaire $(\mathrm{Pd})$;

- la surface totale diaphysaire (STd) ;

- la surface médullaire (SMd) ;

- la surface de la corticale (SCd), calculée par différence des 2 paramètres précédents ;
- le pourcentage de surface corticale par rapport à la surface totale

$$
\% \text { SCd }=\text { SCd } \times 100 / \text { STd ; }
$$

- le facteur de forme FF $=4 \mathrm{p} \times \mathrm{SCd} / \mathrm{Pd}^{2}$;

- la hauteur de la zone mince (ZMd) et de la zone épaisse (ZEd) appréciée par la moyenne de 5 mesures de hauteur effectuées dans chaque zone.

La vitesse d'apposition osseuse, mineralisation apposition rate (MAR), a été mesurée sur les lames examinées en fluorescence. Elle correspond à la moyenne des mesures de l'épaisseur du tissu osseux situé entre les 2 lignes de fluorescence des ostéones en formation. L'épaisseur a été appréciée sur 25 ostéones différents d'une même zone de mesure en réalisant 2 mesures perpendiculaires par ostéone, soit un total de 50 mesures par zone étudiée. La vitesse d'apposition a été calculée en zone mince (ZMd) et en zone épaisse (ZEd). Elle est rapportée à une unité de temps et exprimée en $\mu \mathrm{m} / \mathrm{j}$. 
La fiabilité des mesures a été estimée en calculant le coefficient de variation de 10 mesures effectuées sur le même prélèvement par le même manipulateur dans un temps donné puis de 2 fois 10 mesures espacées dans le temps.

Le coefficient de variation de tous les paramètres cités précédemment ne dépasse pas $6 \%$, soit une variabilité conforme à celle d'un paramètre biologique.

La mesure du coefficient de variation nous a, en revanche, conduits à éliminer de l'étude différents paramètres prévus initialement mais dont le coefficient de variation atteignait parfois 20 à $30 \%$ selon l'âge ou la souche envisagés. C'est le cas notamment de la mesure de la surface de la zone active dont les limites internes sont trop imprécises pour permettre une bonne reproductibilité ou de celle du pourcentage de la surface des canaux vasculaires en zone active. Pour des rajsons analogues, divers paramètres appréciés en fluorescence n'ont pas été conservés tels que :

- le périmètre moyen des ostéones, soit la moyenne des périmètres interne et externe visualisés par les lignes de fluorescence concentriques des ostéones doublement marqués ;

- la surface moyenne des ostéones, c'est-à-dire la surface de tissu osseux compris entre les 2 lignes de fluorescence des ostéones doublement marqués ;

- la surface de la zone sous-périostée qui correspond au territoire situé entre les 2 lignes de fluorescence, concentriques à la diaphyse visualisant les 2 injections de fluorochrome.

Aucune de ces mesures, réalisées à l'aide d'un réticule situé dans l'oculaire du microscope à fluorescence, n'est répétable lorsque l'on fait varier, même de manière minime, l'emplacement de la mesure.

Par ailleurs la mesure de l'épaisseur de la zone sous-périostée s'est révélée techniquement impraticable, la totalité de son épaisseur n'étant pas observable au grossissement du microscope utilisé.

\section{Analyse statistique}

Les valeurs ont été traitées par le logiciel Stat View en utilisant la méthode d'analyse des variances (Anova).

\section{RÉSULTATS}

\section{Poids des animaux et longueur du tibia (tableau II)}

Les différences de poids entre les animaux des 2 souches à un âge donné ou dans une même souche à différents âges sont significatives, avec un accroissement notable de la différence de poids entre les 2 souches à partir de 6 semaines.

II existe également une différence significative de la croissance en longueur du tibia entre les âges et les souches, l'augmentation de la taille du rayon osseux avec l'âge demeurant sensiblement parallèle dans les 2 souches.

\section{Étude histologique qualitative}

La taille générale de la diaphyse augmente de manière évidente avec l'âge dans les 2 souches. Le développement de la surface diaphysaire totale s'accompagne cependant d'une diminution concomitante de la surface osseuse corticale au profit de la surface de la médullaire.

La forme générale de la section transversale médiane de la diaphyse varie de manière plus évidente dans la souche lourde : de forme ronde à 3 semaines, elle apparaît nettement ovoïde à partir de la $5^{e}$ semaine avec, dès cet âge, l'individualisation nette d'une zone corticale mince et d'une zone corticale épaisse qui demeurent visibles tout au long de la période d'observation (fig 2).

Dans la souche fermière la diaphyse apparaît toujours plus ronde ou discrètement ovalaire avec, jusqu'à l'âge de 12 sem, une distinction moins évidente entre zone mince et zone épaisse (fig 3). 
Tableau II. Évolution du poids des animaux et de la longueur du tibia.

\begin{tabular}{|c|c|c|c|c|c|c|c|c|}
\hline & \multicolumn{8}{|c|}{ Âges (en sem) } \\
\hline & 3 & 5 & 6 & 7 & 8 & 9 & 10 & 12 \\
\hline \multicolumn{9}{|c|}{ Poids de l'animal (en g) } \\
\hline \multirow[t]{2}{*}{ Souche fermière } & $283^{a}$ & $628^{b}$ & $833^{\mathrm{C}}$ & $1004^{d}$ & $1407^{e}$ & $1574^{\dagger}$ & 20319 & $2494^{\mathrm{h}}$ \\
\hline & \pm 24 & \pm 51 & \pm 71 & \pm 116 & \pm 88 & \pm 140 & \pm 157 & \pm 126 \\
\hline \multirow[t]{2}{*}{ Souche lourde } & $610^{\mathrm{a}}$ & $1411^{b}$ & $2080^{\circ}$ & $2769^{d}$ & $3525^{e}$ & $4819^{f}$ & $5577^{9}$ & $6422^{\mathrm{h}}$ \\
\hline & \pm 47 & \pm 100 & \pm 196 & \pm 340 & \pm 288 & \pm 227 & \pm 129 & \pm 706 \\
\hline \multicolumn{9}{|c|}{ Longueur du tibia $(e n \mathrm{~cm})$} \\
\hline \multirow[t]{2}{*}{ Souche fermière } & $6,9^{a}$ & $9,2^{b}$ & $10,3^{c}$ & $11,3^{d}$ & $12,8^{e}$ & $13,5^{f}$ & $14,7^{\mathrm{g}}$ & $16,1^{h}$ \\
\hline & $\pm 0,2$ & $\pm 0,2$ & $\pm 0,3$ & $\pm 0,5$ & $\pm 0,5$ & $\pm 0,5$ & $\pm 0,4$ & $\pm 0,4$ \\
\hline \multirow[t]{2}{*}{ Souche lourde } & $8,7^{a}$ & $12^{b}$ & $13,7^{c}$ & $15,3^{d}$ & $16,6^{e}$ & $18,4^{f}$ & $19,1 \mathrm{~g}$ & $20,9^{h}$ \\
\hline & $\pm 0,2$ & $\pm 0,2$ & $\pm 0,7$ & $\pm 0,5$ & $\pm 0,6$ & $\pm 0,4$ & $\pm 0,3$ & $\pm 0,3$ \\
\hline
\end{tabular}

Les moyennes d'une même ligne suivies de 2 indices différents sont significativement différentes (ANOVA, $P<$ $0,05)$; à un âge donné les différences entre les 2 souches sont toujours significatives (ANOVA, $P<0,05$ ).

La position des zones mince et épaisse a été déterminée en utilisant le système de cadran décrit dans le protocole.

La zone la plus mince de la corticale apparaît située dans les 2 souches en position cranio-latérale quel que soit l'âge des animaux. Des exceptions sont constatées chez environ $15 \%$ des animaux des 2 souches à 3 et 8 sem avec des zones minces situées en position caudale ou latérale.

La position de la zone la plus épaisse est plus difficile à apprécier surtout dans la souche fermière. Elle apparaît dans les 2 souches situées dans environ $60 \%$ des cas en position caudo-latérale dans la zone de l'artère nourricière. Dans les autres cas elle se localise en région caudale.

L'analyse histologique de la corticale diaphysaire révèle l'existence de 2 couronnes concentriques, facilement discernables à l'examen au faible grossissement du microscope:
- une zone externe, périphérique, dite "zone active», constituée de nombreux ostéones primaires en formation dont les canaux vasculaires sont de taille importante et qui confèrent à cette zone un aspect lacunaire et poreux ;

- une zone interne de tissu osseux fibrolamellaire d'aspect plus dense, constituée d'ostéones à faible diamètre interne dont la formation est achevée et où se produisent des phénomènes de remodelage.

La zone active est bien visible dès l'âge de 3 sem dans la souche lourde où elle présente une épaisseur importante ; elle s'individualise plus progressivement chez les dindons de la souche fermière où elle devient nettement identifiable seulement vers 5 ou $6 \mathrm{sem}$. Son épaisseur a tendance à diminuer avec l'âge mais elle demeure cependant encore bien visible dans les 2 souches à 12 sem.

Après coloration par le trichrome de Goldner, les canaux vasculaires de la zone active apparaissent bordés par une cou- 


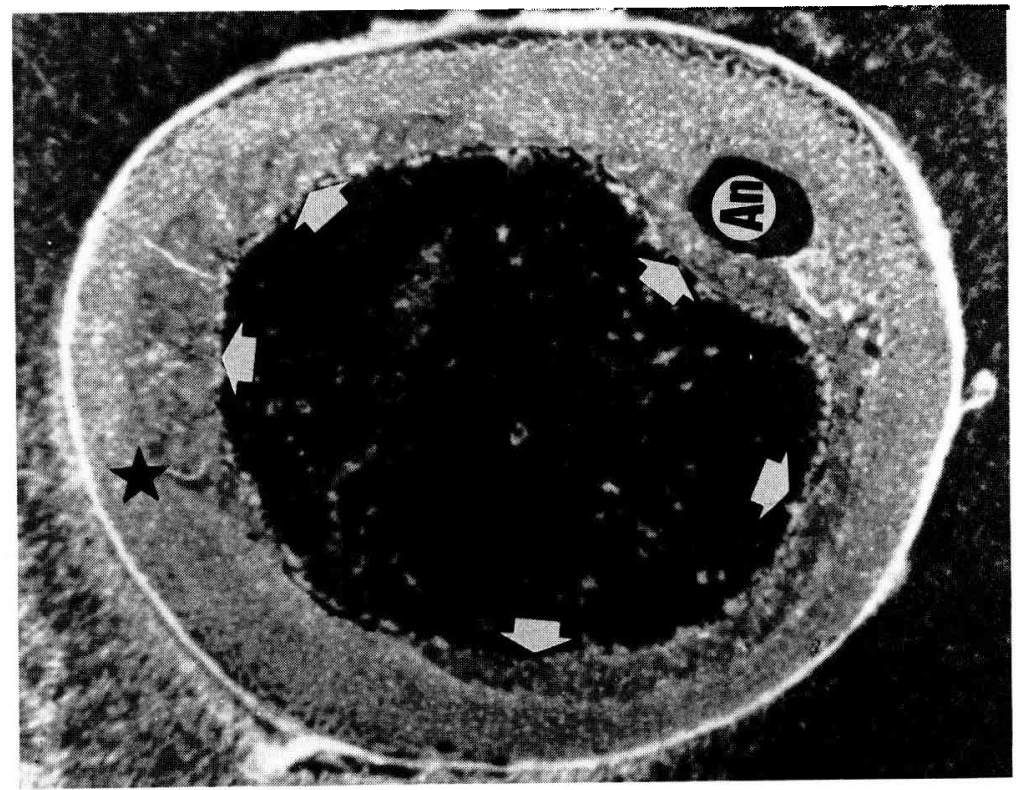

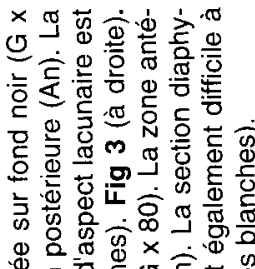

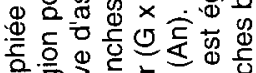

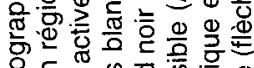

융

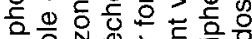

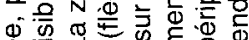

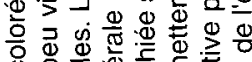

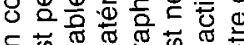

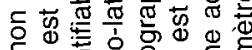

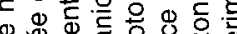

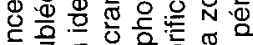

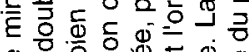

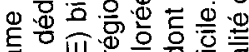

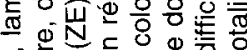

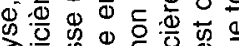

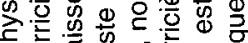

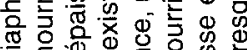

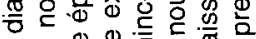

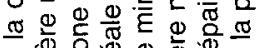

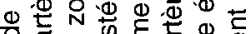

西

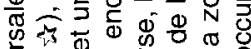

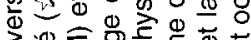

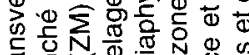

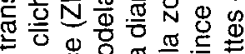

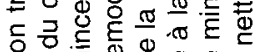

은

ஸ 凹

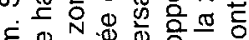

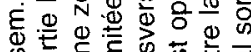

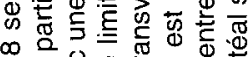

$\infty$ 品

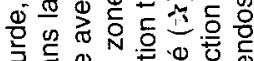

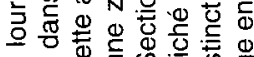

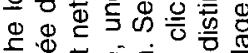

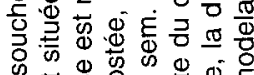

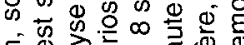

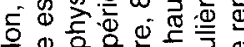

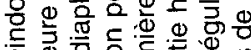

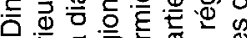

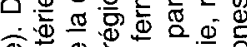

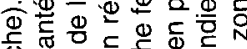
웡 0 ฮั ธั

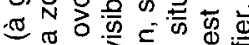

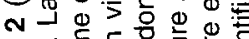

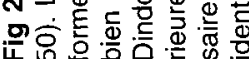


ronne d'ostéoblastes avec un liséré de substance ostéoïde pourpre qui tranche sur la couleur verte de la substance osseuse calcifiée.

L'observation en fluorescence montre, dans la zone active, 2 lignes de fluorescence, concentriques par rapport à la diaphyse, qui correspondent au front de calcification du périoste au moment de l'injection du fluorochrome. La ligne la plus interne correspond à la première injection et la ligne plus externe, située au contact du périoste, à la deuxième injection (fig 4 et 5). Cette zone sous-périostée, délimitée par les 2 lignes concentriques de fluorescence, correspond à la quantité de tissu osseux élaboré par le périoste durant la période d'une semaine séparant les 2 injections de fluorochrome.

Les ostéones marqués par le fluorochrome sont situés essentiellement dans la zone active externe confirmant que l'élaboration du tissu osseux est uniquement périostée.
Fig 4. Dindon, souche lourde, 7 sem. Lame mince de diaphyse examinée en fluorescence dans la région de la zone épaisse ( $\mathrm{G} \times 120)$. La disposition des canaux vasculaires est de type réticulé. (1) Périphérie de la corticale. (2) Ligne de fluorescence externe périostée $\left(2^{e}\right.$ injection de fluorochrome). (3) Ligne de fluorescence interne à disposition sinueuse et irrégulière ( $1^{\text {re }}$ injection de fluorochrome). (4) Ostéones situés entre les 2 lignes du front de calcification avec une seule ligne de marquage interne. (5) Ostéones doublement marqués situés sous la ligne de fluorescence interne. (6) Ostéones à ligne de marquage externe situés en région profonde de la zone active.

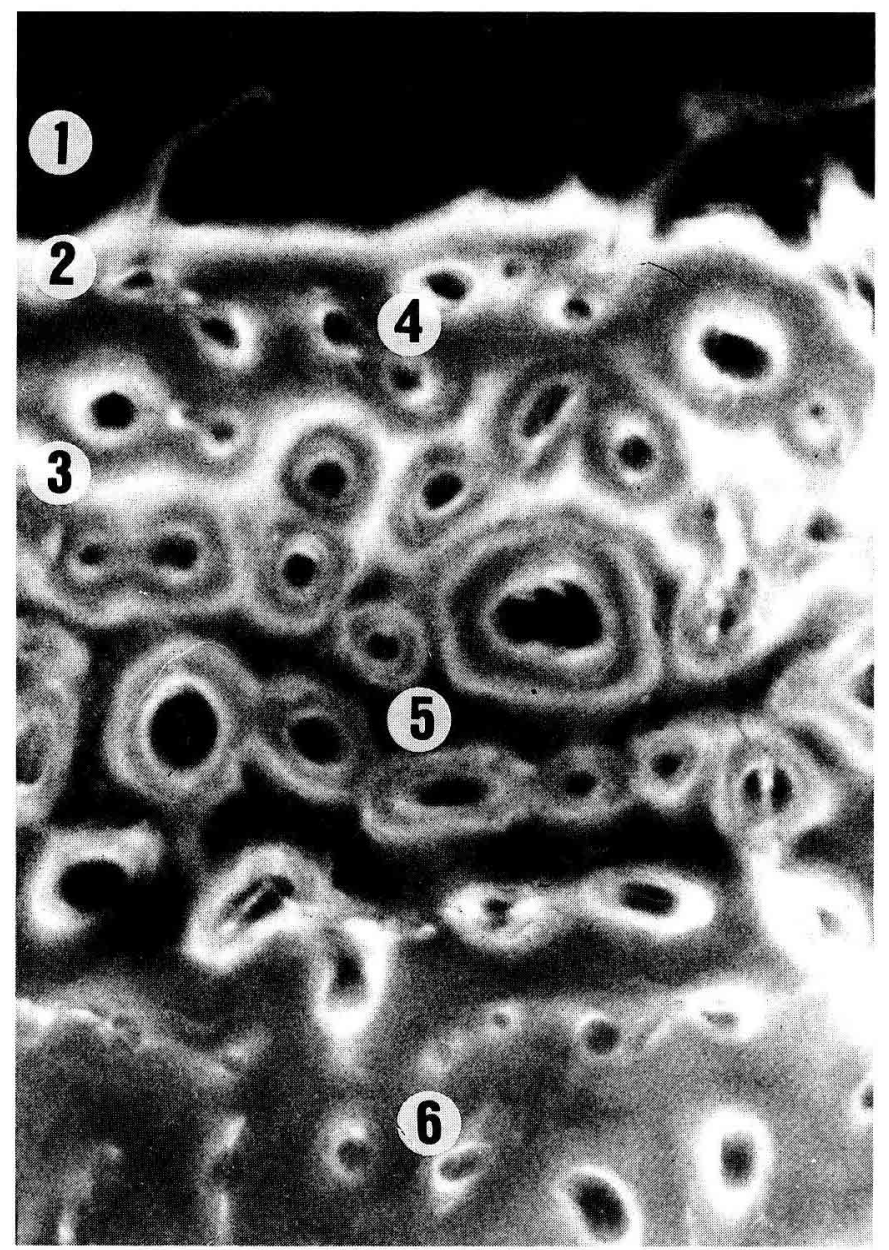




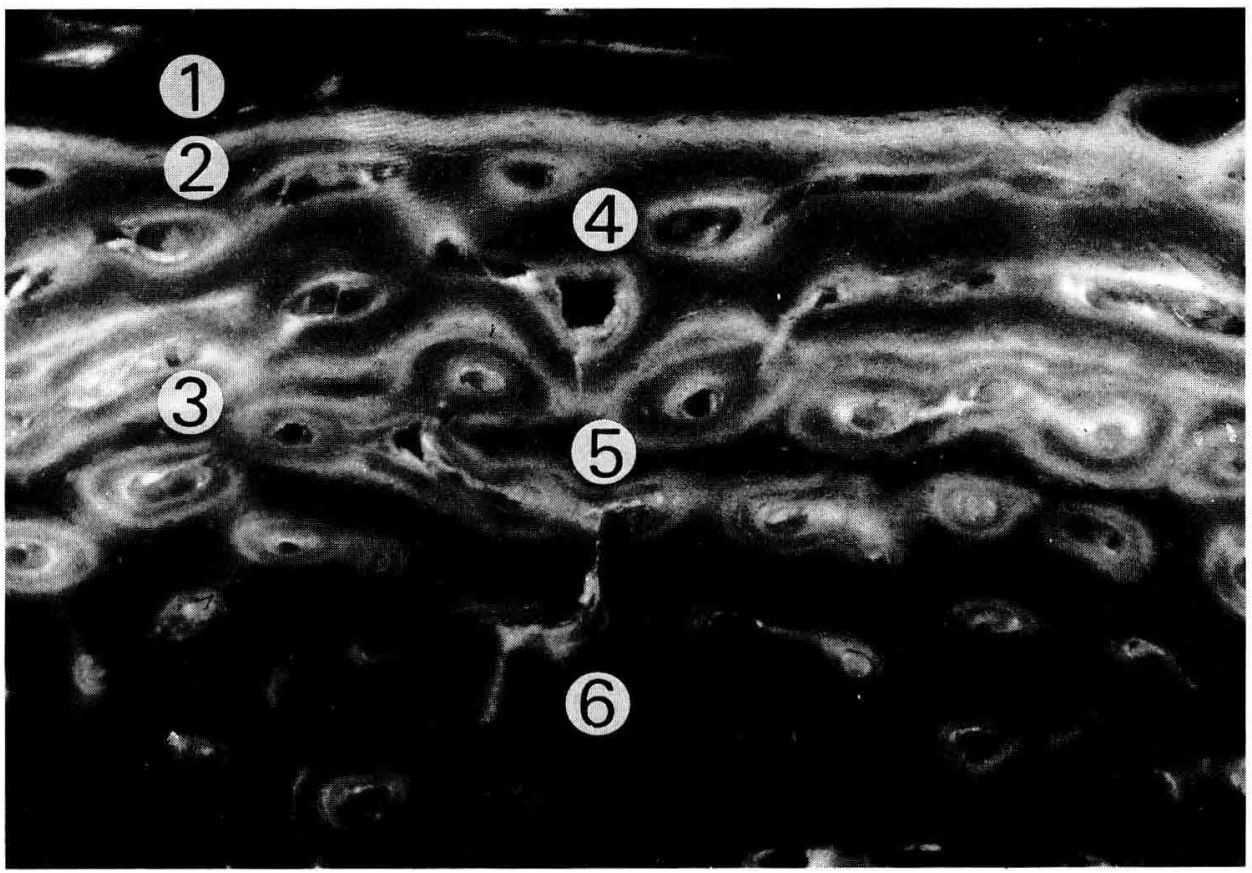

Fig 5. Dindon, souche lourde, 7 sem. Lame mince de diaphyse examinée en fluorescence dans la région de la zone mince $(G \times 120)$. La disposition des canaux vasculaires est de type laminaire. (1) Périphérie de la corticale. (2) Ligne de fluorescence externe, périostée. (3) Ligne de fluorence interne. (4) Ostéones à ligne de marquage interne. (5) Ostéones doublement marqués. (6) Ostéones (peu nombreux) à ligne de marquage externe.

Plusieurs types d'ostéones marqués sont visibles. Les ostéones situés en position interne par rapport à la ligne de fluorescence périostée la plus profonde présentent un double marquage concentrique. L'élaboration des lamelles osseuses étant centripète, la ligne de fluorescence la plus externe correspond à la première injection et la ligne la plus interne à la deuxième injection. L'existence d'un double marquage indique que le temps nécessaire à la constitution des ostéones est supérieur à une semaine (figs 4 et 5 ).

Les ostéones situés plus profondément par rapport aux ostéones doublement marqués ne possèdent que la ligne de fluorescence la plus externe, marquage unique qui indique que la croissance des ostéones s'est achevée avant la deuxième injection du fluorochrome (fig 4).

Les ostéones situés entre les 2 lignes concentriques de marquage périostées ne présentent qu'une ligne de marquage interne, la formation des ostéones de la zone sous-périostée ayant débuté après la première injection de fluorochrome (figs 4 et 5).

Les 2 lignes de marquage et les différents types d'ostéones marqués se retrouvent dans les 2 souches à tous les âges étudiés.

Les proportions relatives des différentes zones différent seulement à 3 sem : dans la souche fermière, la zone des ostéones doublement marqués est presque inexis- 
tante à cet âge et la zone sous périostée est peu épaisse, ne représentant pas plus de $1 / 5$ de l'épaisseur totale de la corticale.

Au contraire, les animaux de la souche lourde possèdent à 3 sem une zone active très développée ; la zone d'ostéones doublement marqués est nettement individualisée et la zone sous-périostée représente plus de la moitié de la hauteur de la corticale, témoignant d'une synthèse osseuse très importante.

Dès la $5^{e}$ semaine l'aspect et les proportions des différents territoires contenant des ostéones marqués apparaissent identiques à un âge donné dans les 2 souches.

L'aspect et la disposition des axes conjonctivo-vasculaires de la zone active diffèrent selon leur localisation dans la diaphyse.

Au niveau de la zone mince, en position cranio-latérale, les canaux vasculaires sont dans les 2 souches à disposition de type laminaire. Ils constituent des canaux circulaires ou allongés disposés en nappes concentriques par rapport à la périphérie de la corticale et présentent peu d'anastomoses entre eux (fig 5).

Au niveau de la zone épaisse, en position caudale ou caudo-latérale, les canaux vasculaires présentent soit une structure réticulée formant des cavités à disposition irrégulière, non ordonnée, très largement anatomosées entre elles (fig 4), soit une disposition rayonnante ou radiée avec des canaux vasculaires peu anastomosés entre eux et à disposition perpendiculaire par rapport à la périphérie de la corticale.

Dans la souche fermière, la zone épaisse moins nettement identifiable présente majoritairement des canaux vasculaires de structure réticulée.

Dans la souche lourde où la zone épaisse est mieux individualisée, une disposition rayonnante domine à tous les âges et devient même exclusive à partir de 9-12 sem.

La zone interne de la corticale diaphysaire est constituée d'un tissu fibrolamellaire dont les canaux vasculaires sont de faible diamètre, à disposition longitudinale dispersée, séparés les uns des autres par un abondant tissu ossifié. L'absence d'ostéoblastes et de substance ostéoïde après coloration par le trichrome de Goldner et celle de zones de marquage en fluorescence montrent que la synthèse osseuse est achevée à leur niveau.

La zone interne est tout d'abord d'importance réduite, notamment chez les animaux de souche lourde âgés de 3 sem, puis sa surface augmente progressivement avec l'âge aux dépens de celle de la zone active périphérique dont l'importance régresse au cours du temps.

Des phénomènes de remodelage apparaissent également en région endostéale. Ils se traduisent par la présence de lacunes irrégulières occupées par des canaux vasculaires qui confèrent à l'endoste un aspect déchiqueté.

Le trichrome de Goldner révèle la présence d'ostéoclastes plurinucléés associés à une assise de cellules ostéoblastiques situées au contact d'un liséré de substance ostéoïde pourpre. L'examen en fluorescence confirme l'existence d'une activité de synthèse osseuse en révélant dans les zones de remodelage des ostéones en formation doublement marqués par le fluorochrome (fig 6).

Les zones de remodelage endostéales apparaissent dès la $5^{\mathrm{e}}$ semaine dans les 2 souches (figs 2 et 3 ). Elles sont plus marquées dans la souche fermière où le phénomène apparaît plus étendu et atteint un nombre plus élevé d'animaux. Le phénomène se localise préférentiellement dans la zone mince cranio-latérale (fig 2), cependant dans les cas d'une atteinte plus importante le remodelage progresse en di- 


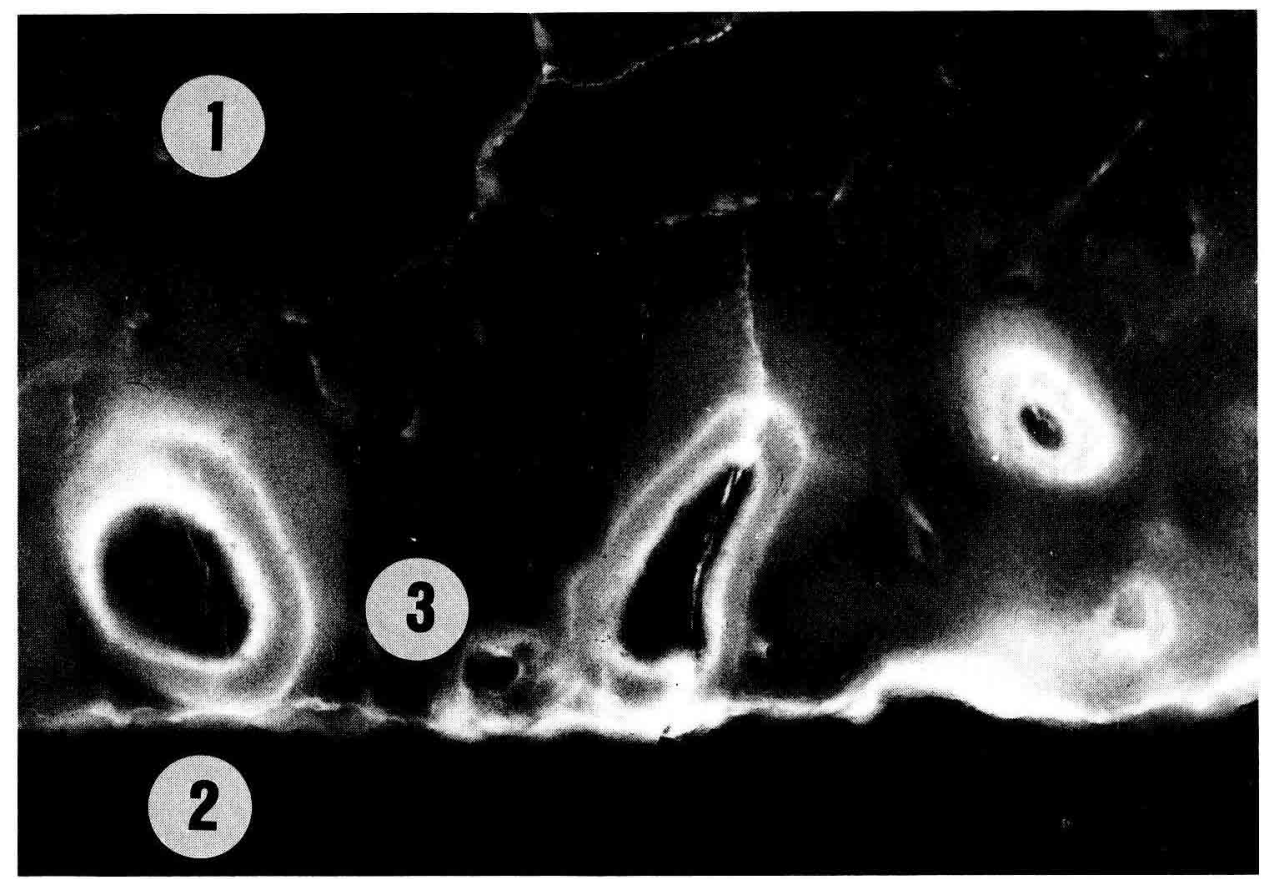

Fig 6. Dindon, souche fermière, 12 sem. Lame mince de diaphyse examinée en fluorescence en région endostéale. (1) Zone interne de la corticale, absence d'ostéones en formation, marqués par le fluorochrome. (2) Cavité méduliaire et endoste. (3) Ostéones secondaires doublement marqués de la zone de remodelage endostéal.

rection de la zone caudo-latérale où est située l'artère nourricière et peut s'étendre chez certains animaux à la presque totalité de la face endostéale (fig 3 ).

\section{Étude histomorphométrique}

Les résultats des paramètres histomorphométriques mesurés dans les 2 souches figurent dans les tableaux III et IV et les figures 7 et 8 .

On constate dans les 2 souches, entre 3 et 12 sem, une augmentation constante du périmètre $(\mathrm{Pd})$ et des surfaces diaphysaires, qu'il s'agisse de la surface totale (STd) ou de celles de la corticale (SCd) et de la médullaire (SMd). Cependant, il n'existe pas toujours de différences significatives entre les animaux d'une même souche à 2 âges successifs notamment chez les animaux les plus âgés ( 8 à 12 sem).

La croissance générale de la diaphyse apparaît relativement régulière et linéaire dans la souche fermière ; elle présente par contre une accélération dans la souche lourde vers l'âge de 7-8 sem, âge où la croissance pondérale s'accélère également dans cette souche (fig 7).

Le pourcentage de surface corticale diminue régulièrement avec le temps dans les 2 souches. La différence entre 2 âges n'est pas statistiquement significative dans la souche fermière durant toute la période 
Tableau III. Paramètres histomorphométriques: périmètre, surfaces diaphysaires et facteur de forme.

\begin{tabular}{|c|c|c|c|c|c|c|c|c|}
\hline & & & & $\hat{A} g e s$ & (en sem) & & & \\
\hline & 3 & 5 & 6 & 7 & 8 & 9 & 10 & 12 \\
\hline Périmètre diaphysá & aire $(P d)(\epsilon$ & $n \mathrm{~mm})$ & & & & & & \\
\hline Souche fermière & $12,6^{a}$ & $16,8^{b}$ & $19^{c}$ & $20,4^{d}$ & $23,8^{e}$ & $25,6^{\mathrm{e}}$ & $27,2^{f}$ & 29,59 \\
\hline & $\pm 0,8$ & $\pm 0,7$ & \pm 1 & $\pm 0,8$ & $\pm 1,2$ & $\pm 2,8$ & $\pm 1,8$ & $\pm 3,4$ \\
\hline Souche lourde & $16,8^{a}$ & $24,6^{b}$ & $27,7^{c}$ & $30,1^{d}$ & $34,6^{\mathrm{e}}$ & $38,7^{f}$ & 41,89 & 44,69 \\
\hline & $\pm 0,7$ & $\pm 1,2$ & $\pm 1,5$ & $\pm 2,8$ & $\pm 1,8$ & $\pm 2,6$ & $\pm 2,5$ & $\pm 1,6$ \\
\hline Surface totale diap & hysaire (S & $T d)(e n m r$ & $\left.m^{2}\right)$ & & & & & \\
\hline Souche fermière & $12^{\mathrm{a}}$ & $21^{\mathrm{b}}$ & $28^{\mathrm{c}}$ & $32^{d}$ & $43^{\mathrm{e}}$ & $51^{\mathrm{e}}$ & $58^{f}$ & $67^{f}$ \\
\hline & \pm 1 & \pm 1 & \pm 3 & \pm 2 & \pm 4 & \pm 12 & \pm 5 & \pm 14 \\
\hline Souche lourde & $21^{a}$ & $46^{\mathrm{b}}$ & $57^{c}$ & $69^{d}$ & $90^{\mathrm{e}}$ & $112^{f}$ & $132^{f}$ & $149^{f}$ \\
\hline & \pm 2 & \pm 4 & \pm 7 & \pm 12 & \pm 8 & \pm 16 & \pm 18 & \pm 10 \\
\hline Surface médullaire & $(S M d)(e r$ & $\left.m m^{2}\right)$ & & & & & & \\
\hline Souche fermière & $4^{a}$ & $8^{\mathrm{b}}$ & $11^{\mathrm{c}}$ & $14^{d}$ & $19^{e}$ & $23^{f}$ & 279 & $35^{h}$ \\
\hline & \pm 1 & \pm 1 & \pm 1 & \pm 2 & \pm 3 & \pm 6 & \pm 3 & \pm 9 \\
\hline Souche lourde & $7^{a}$ & $17^{\mathrm{b}}$ & $25^{\mathrm{c}}$ & $33^{d}$ & $41^{e}$ & $53^{f}$ & $63^{f}$ & $81^{g}$ \\
\hline & \pm 1 & \pm 3 & \pm 4 & \pm 8 & \pm 6 & \pm 8 & \pm 12 & \pm 5 \\
\hline Surface corticale (s & $S C d)(e n n$ & $\left.n m^{2}\right)$ & & & & & & \\
\hline Souche fermière & $7^{a}$ & $13^{b}$ & $16^{c}$ & $17^{d}$ & $23^{e}$ & $27^{e}$ & $30^{f}$ & $32^{f}$ \\
\hline & \pm 1 & \pm 1 & \pm 2 & \pm 2 & \pm 2 & \pm 5 & \pm 2 & \pm 6 \\
\hline Souche lourde & $14^{a}$ & $28^{b}$ & $32^{\mathrm{b}}$ & $35^{\mathrm{c}}$ & $48^{d}$ & $58^{e}$ & $69^{\circ}$ & $68^{\mathrm{e}}$ \\
\hline & \pm 1 & \pm 3 & \pm 3 & \pm 7 & \pm 3 & \pm 7 & \pm 7 & \pm 6 \\
\hline Pourcentage surfac & ce cortical & $e(\% S C d)$ & $(\%)$ & & & & & \\
\hline Souche fermière & $65,1^{\mathrm{a}}$ & $62,2^{\mathrm{a}}$ & $58,2^{\mathrm{a}}$ & $54,9^{\mathrm{a}}$ & $54,8^{\mathrm{a}}$ & $53,8^{a}$ & $52,5^{a}$ & $48,8^{a}$ \\
\hline & $\pm 1,8 \mathrm{NS}$ & $\pm 3,8 \mathrm{NS}$ & $\pm 3,7 \mathrm{NS}$ & $\pm 5,4 \mathrm{NS}$ & $\pm 4,1 \mathrm{NS}$ & $\pm 2,4$ NS & $\pm 3,1 \mathrm{NS}$ & $\pm 4,7 \mathrm{NS}$ \\
\hline Souche lourde & $67,8^{a}$ & $61,5^{\mathrm{b}}$ & $55,7^{c}$ & $51,2^{c}$ & $54,0^{c}$ & $53,3^{c}$ & $52,5^{d}$ & $45,6^{d}$ \\
\hline & $\pm 4,7$ & $\pm 4,3$ & $\pm 4,5$ & $\pm 5,4$ & $\pm 3,0$ & $\pm 1,4$ & $\pm 2,9$ & $\pm 1,9$ \\
\hline Facteur de Forme & $(F F)(\%)$ & & & & & & & \\
\hline Souche fermière & $95,8^{a}$ & $96,7^{a}$ & $96,6^{\mathrm{a}}$ & $97^{\mathrm{a}}$ & $96^{a}$ & $96,4^{\mathrm{a}}$ & $96,3^{a}$ & $96,3^{\mathrm{a}}$ \\
\hline & $\pm 0,7 \mathrm{NS}$ &, \pm 05 NS & $\pm 0,8 \mathrm{NS}$ & $\pm 0,5$ NS & $\pm 0,8 \mathrm{NS}$ & $\pm 0,4$ NS & $\pm 0,7 \mathrm{NS}$ & $\pm 1,3 \mathrm{NS}$ \\
\hline Souche lourde & $96,1^{\mathrm{a}}$ & $95,7^{\mathrm{a}}$ & $95,1^{a}$ & $94,7^{\mathrm{a}}$ & $94^{\mathrm{a}}$ & $93,7^{\mathrm{a}}$ & $93,5^{\mathrm{a}}$ & $94^{a}$ \\
\hline & $\pm 1,3$ & $\pm 0,6$ & $\pm 0,1$ & $\pm 0,9$ & $\pm 1,1$ & $\pm 0,1$ & $\pm 1,7$ & $\pm 1,2$ \\
\hline
\end{tabular}

Les moyennes d'une même ligne suivies de 2 indices différents sont significativement différentes (ANOVA, $P<0,05$ ); lorsque la différence entre les 2 souches à un âge donné est non significative, elle est notée NS (ANOVA, $P<0,05)$.

d'observation. Elle est par contre significative dans la souche lourde en début de période de croissance ( 3 à 6 sem) et également entre 9 et 10 sem.

Le facteur de forme (FF), calculé à partir des mesures du périmètre $(P d)$ et de la surface totale (STd), permet de quantifier les modifications de forme du fût diaphysaire. II est différent de 100 dans les 2 souches à tous les âges étudiés, indiquant que la forme générale de la diaphyse s'éloigne d'un cercle parfait quel que soit 
Tableau IV. Paramètres histomorphométriques : épaisseurs de la corticale et vitesse d'apposition osseuse.

\begin{tabular}{|c|c|c|c|c|c|c|c|c|}
\hline & & & & $\hat{A} g e$ & (en sem) & & & \\
\hline & 3 & 5 & 6 & 7 & 8 & 9 & 10 & 12 \\
\hline Hauteur de la zone & mince & $Z M d)(e n$ & $m m)$ & & & & & \\
\hline Souche fermière & $0,5^{\mathrm{a}}$ & $0,7^{\mathrm{b}}$ & $0,7^{b}$ & $0,7^{\mathrm{b}}$ & $0,9^{c}$ & $0,8^{c}$ & $0,8^{c}$ & $0,8^{c}$ \\
\hline & $\pm 0,1$ & $\pm 0,1$ & $\pm 0,1$ & $\pm 0,1$ & $\pm 0,1 \mathrm{~ns}$ & $\pm 0,1$ & $\pm 0,1$ & $\pm 0,1$ \\
\hline Souche lourde & $0,7^{a}$ & $0,9^{b}$ & $0,9^{b}$ & $1^{b}$ & $1^{\mathrm{b}}$ & $1,1 b$ & $1^{b}$ & $1,1^{b}$ \\
\hline & $\pm 0,1$ & $\pm 0,1$ & $\pm 0,1$ & $\pm 0,1$ & $\pm 0,1$ & $\pm 0,1$ & $\pm 0,1$ & $\pm 0,1$ \\
\hline Hauteur de la zone & épaisse & $(Z E d)(e n$ & $\mathrm{mm})$ & & & & & \\
\hline Souche fermière & $1^{\mathrm{a}}$ & $1,2^{\mathrm{b}}$ & $1,2^{\mathrm{b}}$ & $1,2^{b}$ & $1,5^{\mathrm{c}}$ & $1,6^{\mathrm{C}}$ & $1,6^{\mathrm{C}}$ & $1,7^{c}$ \\
\hline & $\pm 0,1$ & $\pm 0,1$ & $\pm 0,1$ & $\pm 0,2$ & $\pm 0,2$ & $\pm 0,21$ & $\pm 0,2$ & $\pm 0,3$ \\
\hline Souche lourde & $1,5^{\mathrm{a}}$ & $1,8^{b}$ & $1,6^{b}$ & $1,8^{b}$ & $2,1^{\mathrm{c}}$ & $2,3^{c}$ & $2,6^{c}$ & $2,2^{c}$ \\
\hline & $\pm 0,2$ & $\pm 0,3$ & $\pm 0,2$ & $\pm 0,, 5$ & $\pm 0,2$ & $\pm 0,3$ & $\pm 0,1$ & $\pm 0,3$ \\
\hline Vitesse apposition & osseuse & zone $\min$ & $c e(M A R / Z$ & $M d)(e n \mu r$ & & & & \\
\hline Souche fermière & & $2,32^{\mathrm{a}}$ & $2,50^{\mathrm{b}}$ & $2,34^{\mathrm{b}} \mathrm{ns}$ & $2,99^{\circ} \mathrm{ns}$ & $4,69^{d}$ & $2,87^{e}$ & $3,06^{\mathrm{e}}$ \\
\hline & & $\pm 0,10$ & $\pm 0,13$ & $\pm 0,18$ & $\pm 0,19 \mathrm{~ns}$ & $\pm 0,46$ & $\pm 0,24 \mathrm{~ns}$ & $\pm 0,33 \mathrm{~ns}$ \\
\hline Souche lourde & $2,69^{a}$ & $2,70^{a}$ & $2,75^{a}$ & $2,88^{a}$ & $2,92^{\mathrm{a}}$ & $3,10^{\mathrm{a}}$ & $2,82^{a}$ & $2,94^{a}$ \\
\hline & $\pm 0,37$ & $\pm 0,14$ & $\pm 0,26 \mathrm{~ns}$ & $\pm 0,30$ & $\pm 0,18 \mathrm{~ns}$ & $\pm 0,27 \mathrm{~ns}$ & $\pm 0,11 \mathrm{~ns}$ & $\pm 0,10$ \\
\hline Vitesse d'appositio & nosseus & se zone ép & baisse (MA & $R / S E d)(\mu r$ & & & & \\
\hline Souche fermière & & $2,63^{a}$ & $2,66^{\mathrm{a}}$ & $2,56^{a}$ & $3,15^{\mathrm{b}}$ & $5,41^{c}$ & $3,44^{b}$ & $3,62^{b}$ \\
\hline & & $\pm 0,14$ & $\pm 0,15 \mathrm{~ns}$ & $\pm 0,37$ & $\pm 0,12 \mathrm{~ns}$ & $\pm 0,59$ & $\pm 0,30 \mathrm{~ns}$ & $\pm 0,25$ \\
\hline Souche lourde & 2,90 & 2,90 & 2,87 & 3,08 & 3,03 & 3,32 & 3,23 & 3,28 \\
\hline & $\pm 0,21^{a}$ & $\pm 0,19^{a}$ & $\pm 0,26^{\mathrm{a}}$ & $\pm 0,21^{a}$ & $\pm 0,31^{a}$ & $\pm 0,34^{b}$ & $\pm 0,07^{b}$ & $\pm 0,12^{b}$ \\
\hline
\end{tabular}

Les moyennes d'une même ligne suivies de 2 indices différents sont significativement différentes (ANOVA, $P<$ $0,05)$; lorsque la différence entre les 2 souches à un âge donné est non significative, elle est notée NS (ANOVA, $P<$ $0,05)$; lorsque la différence, dans une même souche, entre la zone mince et la zone épaisse n'est pas significante elle est notée ns (ANOVA, $P<0,05$ ). * Paramètre non mesuré en raison du trop faible nombre d'oestéones doublement marqués.

l'âge des animaux. II n'apparaît pas d'évolution significative du facteur de forme entre 2 âges consécutifs dans aucune des 2 souches.

Dans une même souche, la différence de hauteur entre la zone épaisse (ZEd) et la zone mince ( $Z M d)$ de la cortical est significative à tous les âges étudiés (fig 8).

La croissance diaphysaire s'accompagne d'une augmentation régulière mais modérée de l'épaisseur de la corticale dans la zone épaisse et la zone mince, les différences entre 2 âges consécutifs étant rarement significatives.

La vitesse d'apposition osseuse (MAR) varie peu avec l'âge aussi bien en zone mince qu'en zone épaisse. Ce paramètre est toutefois plus variable dans la souche fermière avec une accélération du phénomène entre 7 et 10 sem quelle que soit la zone considérée.

La comparaison des paramètres entre les 2 souches à un âge donné montre que le périmètre $(\mathrm{Pd})$ et les surfaces diaphy- 


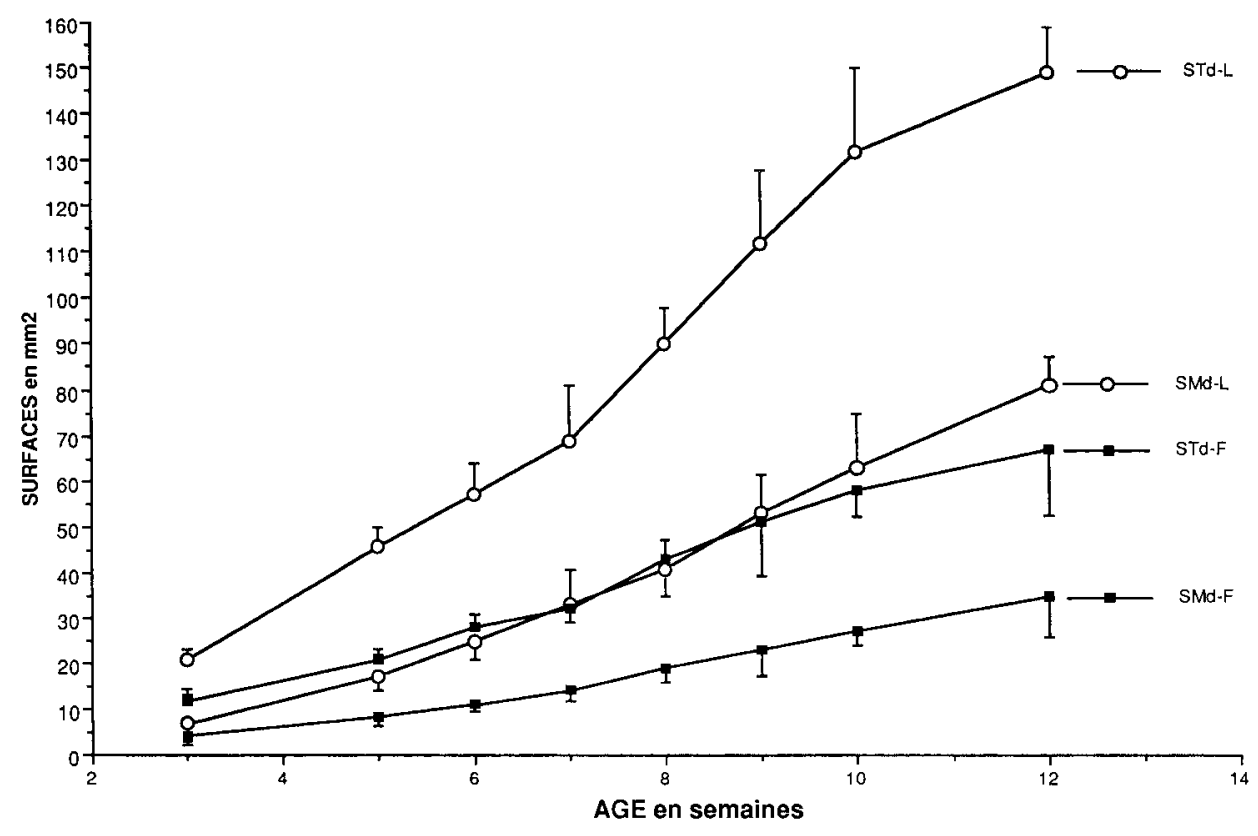

Fig 7. Évolution de la surface diaphysaire totale (STd) et de la surface médullaire (SMd) avec l'âge. STd-L : surface diaphysaire totale, souche lourde. SMd-L : surface médullaire, souche lourde. STd$F$ : surface diaphysaire totale, souche fermière. SMd-F : surface médullaire, souche fermière.

saires (STd, SMd et SCd) sont toujours significativement supérieurs dans la souche lourde à tous les âges de notre étude (fig 7). Par contre le pourcentage de surface corticale (\% SCd) et le facteur de forme (FF) ne sont pas statistiquement différents entre les 2 souches, quel que soit l'âge.

À l'exception des animaux de 8 sem pour la zone mince, l'épaisseur de la corticale est, dans les 2 zones, toujours statistiquement supérieure dans la souche lourde (fig 8).

La vitesse d'apposition osseuse n'a pas été mesurée à 3 sem dans la souche fermière en raison du trop faible nombre d'ostéones doublement marqués. La compa- raison de ce paramètre entre les 2 souches varie selon l'âge. La vitesse d'apposition est plus rapide dans la souche lourde chez les jeunes animaux; par contre elle devient plus rapide dans la souche fermière à 9 sem et demeure toujours statistiquement plus élevée à $12 \mathrm{sem}$ en zone épaisse pour cette même souche.

\section{DISCUSSION}

Les descriptions histologiques de la croissance osseuse diaphysaire sont peu nombreuses chez les oiseaux et se rapportent essentiellement au poulet (Dammrich et Rodenhoff, 1970 ; Rodenhoff et Dammrich, 


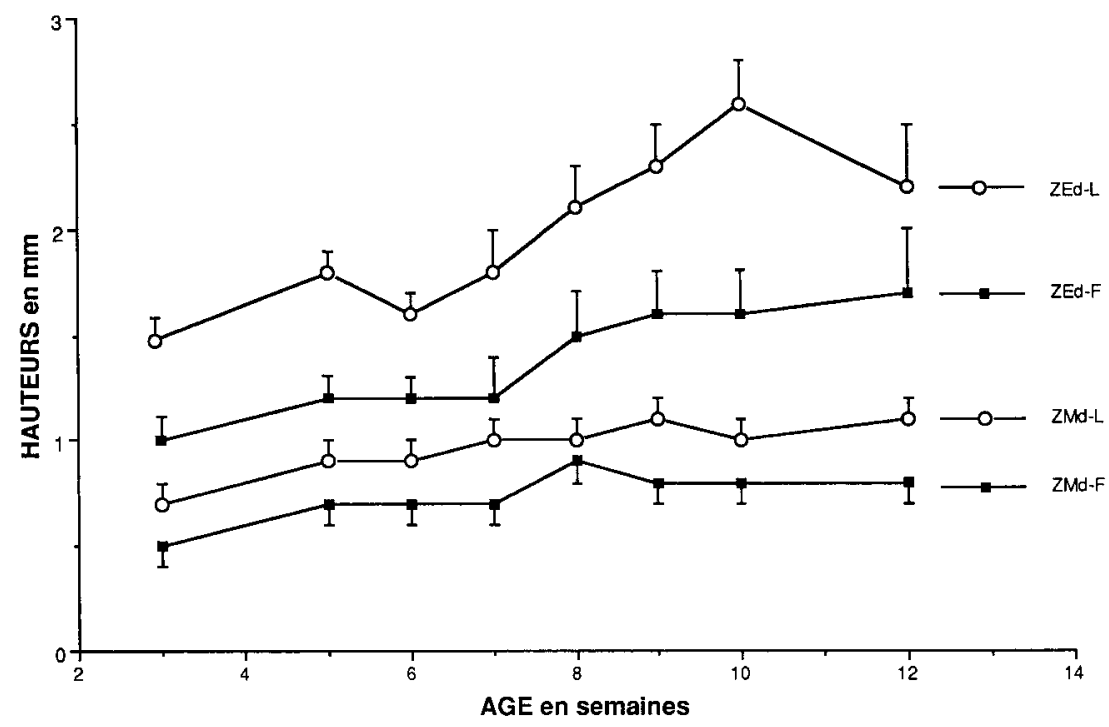

Fig 8. Évolution des hauteurs de la zone mince (SMd) et de la zone épaisse (ZEd) avec l'âge. ZEd-L : zone épaisse, souche lourde. ZMd-L : zone mince, souche lourde. ZEd-F : zone épaisse, souche fermière. $Z M d-F$ : zone mince, souche fermière.

1971 ; Shandu et Jande, 1981 ; Ranaweera et Wise 1981, Pellegrino et Biltz 1983; Leterrier et Nys, 1992a).

Une étude préalable du tissu osseux de la dinde a montré l'intérêt des méthodes histologiques et histomorphométriques pour comparer les caractéristiques de la croissance osseuse dans 2 souches de dinde différentes par leur poids et leur vitesse de croissance (Leblanc et al, 1986). Les différences précoces de la croissance diaphysaire ont été confirmées dans une étude ultérieure chez des animaux âgés de 1 j à 6 sem (Wyers et al, 1993).

Nous avions pour but de compléter la comparaison de la croissance diaphysaire des 2 mêmes souches de dinde par l'étude d'animaux âgés de 3 à 12 sem. Cette période correspond à la phase de croissance la plus rapide au cours de laquelle peuvent éventuellement apparaître des particulari- tés du développement osseux susceptibles d'expliquer l'évolution clinique de troubles locomoteurs dans les souches lourdes à partir de 10-12 sem (Chérel et al, 1991a).

Nos résultats confirment que, chez la dinde (Leblanc et al, 1986 ; Wyers et al, 1993) comme chez le poulet (Pratt, 1961; Sandhu et Jande, 1981 ; Leterrier et Nys, 1992a), la croissance diaphysaire est essentiellement périostéale avec la distinction précoce d'une zone active périphérique où se forment les nouveaux ostéones. La zone interne endostéale est uniquement le siège de phénomènes de remodelage (Pratt, 1961; Sandhu et Jande, 1981 ; Pellegrino et Biltz, 1983 ; Wyers et al,1993).

La synthèse osseuse apparaît plus précoce et plus intense dans la souche lourde comme en témoigne l'apparition d'une zone active épaisse et nettement indivi- 
dualisée dès la $3^{\text {e }}$ sem d'âge alors que celle-ci demeure difficile à identifier dans la souche fermière avant 5 ou 6 sem. L'examen en fluorescence confirme par ailleurs l'abondance des ostéones doublement marqués dans la zone active dès l'âge de $3 \mathrm{sem}$, dans la souche lourde, alors que ceux-ci demeurent trop peu nombreux pour apprécier la vitesse d'apposition osseuse chez les animaux fermiers de même âge.

En relation avec une activité de synthèse osseuse plus précoce dans la souche lourde, le pourcentage de surface corticale est plus élevé dans cette souche jusqu'à l'âge de 2 sem (Wyers et al, 1993). Dès l'âge de 3 sem, le pourcentage de surface corticale apparaît cependant comparable dans les 2 souches et le demeure durant toute la période de notre observation. Ce paramètre augmente dans les 2 souches jusqu'à 2 sem puis a tendance à diminuer régulièrement jusqu'à 12 sem.

II ne nous a pas été possible, avec le matériel utilisé pour notre étude, de mesurer dans des conditions de reproductibilité satisfaisante divers paramètres quantifiant l'activité de synthèse osseuse du périoste notamment après marquage en fluorescence. La structure fibro-lamellaire du tissu osseux des oiseaux ne permet pas, en effet, de compter le nombre absolu ou relatif d'ostéones dans une zone donnée de la corticale ni de mesurer avec précision la surface des canaux vasculaires notamment chez les animaux de souche lourde à partir de 5 à 6 sem. L'intensité de la synthèse périostée ne peut donc être appréciée qu'à travers l'importance et les caractéristiques de la croissance totale de la diaphyse.

La croissance en épaisseur de la corticale diaphysaire est nettement supérieure dans la souche lourde à tous les âges étudiés. Alors que la surface diaphysaire totale apparaissait comparable dans les 2 souches à $1 \mathrm{j}$ et 1 sem, elle devient signifi- cativement supérieure dans la souche lourde dès l'âge de 2 sem (Wyers et al, 1993) pour dépasser le double de la surface de la diaphyse de la souche fermière à l'âge de $5 \mathrm{sem}$. Les écarts de surface entre les 2 souches s'accentuent ensuite avec l'âge parallèlement à l'augmentation beaucoup plus rapide du poids chez les animaux de souche lourde (fig 7). L'augmentation importante de la taille de la diaphyse est associée à une résorption endostéale supérieure dans la souche lourde. Celle-ci se traduit par une augmentation plus rapide de la surface de la médullaire diaphysaire comparativement à celle de la souche fermière (fig 7 ).

Les phénomènes de remodelage en région endostéale semblent, en revanche, beaucoup plus importants dans la souche fermière. À la différence de la souche lourde, de nombreuses images de remodelage sont visibles dans cette souche, en zone mince de la corticale diaphysaire dès la $5^{e}$ sem et la fréquence et l'intensité du phénomène demeurent toujours plus marquées dans la souche fermière quel que soit l'âge considéré.

La résorption osseuse endostéale nettement plus lente dans la souche légère permet, sans doute, une expression plus évidente des capacités de remodelage de la face interne de la diaphyse dans cette souche.

Dans différentes espèces animales, y compris le dindon, les forces mécaniques exercées sur le tissu osseux semblent jouer un rôle prépondérant sur l'apparition et l'intensité des phénomènes de remodelage (Lanyon, 1984). Cette constatation peut paraître en contradiction avec nos résultats qui montrent des phénomènes de remodelage plus précoces et plus marqués dans la souche fermière, apparemment soumise à des contraintes mécaniques moindres. À noter cependant que les résultats expérimentaux publiés ont été obtenus chez des animaux adultes chez qui 
la croissance osseuse est terminée et non chez des animaux en croissance comme ceux de notre étude.

L'augmentation générale de la taille de la région médiane de la diaphyse s'accompagne de modifications de forme de celleci. De forme régulière et ronde à $3 \mathrm{sem}$, la diaphyse devient progressivement ovoïde avec l'individualisation, dans les 2 souches, d'une zone épaisse et d'une zone mince de hauteur significativement différente. La disposition des axes conjonctivovasculaires du tissu fibrolamellaire n'est pas la même selon la zone considérée, différences histologiques qui, selon de Ricqles $(1975,1977)$, correspondent à des vitesses d'élaboration osseuses différentes. Selon cet auteur, l'aspect rayonnant ou réticulé observé en zone épaisse résulte d'une élaboration osseuse de vitesse élevée, alors que l'aspect laminaire, caractéristique de la zone mince, correspond à une vitesse d'élaboration osseuse plus lente associée à une quantité supérieure de tissu osseux élaboré.

Comme constaté précédemment chez les très jeunes animaux (Wyers et al, 1993), des différences de forme existent entre les diaphyses des animaux des 2 souches. La forme ovoïde et l'individualisation d'une zone mince et d'une zone épaisse sont plus précoces et plus évidentes dans la souche lourde; l'aspect rayonnant des canaux vasculaires qui prédomine en zone épaisse chez la plupart des animaux de souche lourde correspondant, selon de Ricqles $(1975,1977)$, à la forme d'élaboration osseuse la plus rapide du tissu fibrolamellaire. Comme le proposent Rodenhoff et Dammrich (1971) chez le poulet ou Frost (1973) chez les Mammifères, la croissance différentielle de la corticale diaphysaire serait la conséquence des contraintes mécaniques importantes exercées précocement sur l'os cortical de la souche lourde dont le poids augmente

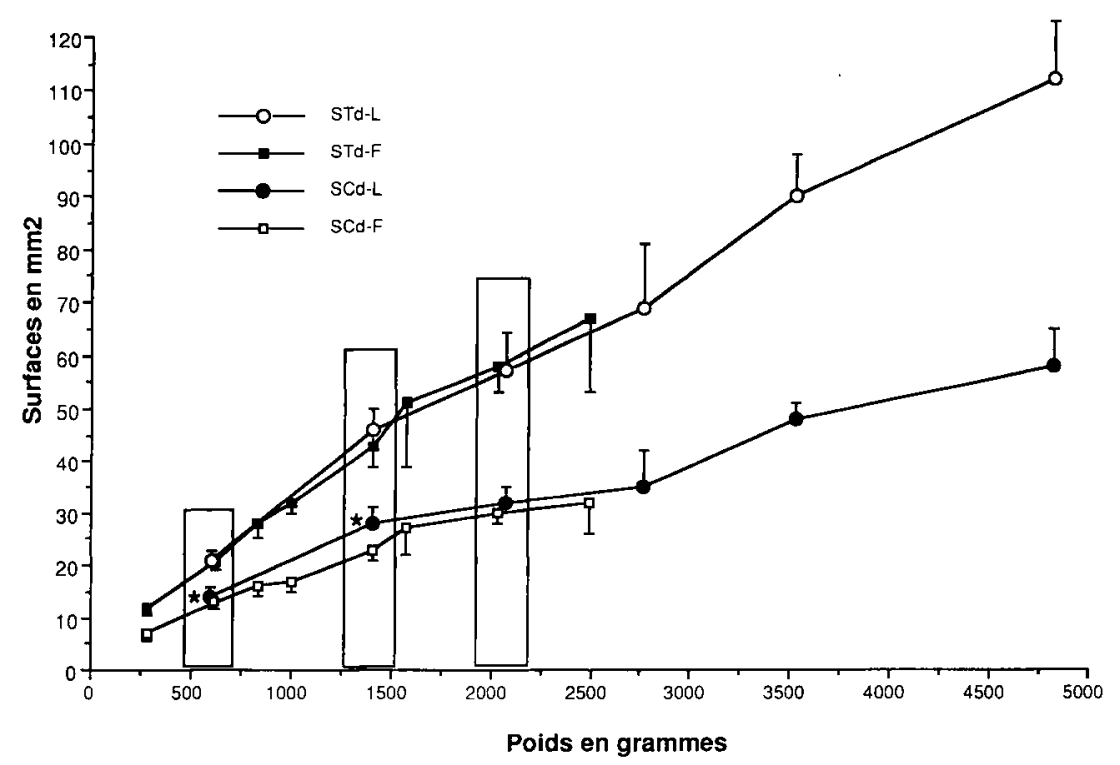

Fig 9. Comparaison de la surface diaphysaire totale (STd) et de la surface corticale (SCd) à poids égal. Les paramètres comparés à poids égal dans les 2 souches sont encadrés. Les paramètres statistiquement différents sont indiqués : *. 
beaucoup plus rapidement que celui de la souche fermière.

Le problème d'une plus grande fragilité de l'os fibro-lamellaire d'élaboration rapide en relation avec une porosité plus grande de ce dernier reste posé. Les travaux de Leterrier et Nys (1992a) sur 2 souches de poulets différentes par leur vitesse de crojsance semblent démontrer une plus grande porosité de la diaphyse associée à une réduction des propriétés mécaniques de flexion de l'os chez les animaux à croissance rapide, plus sujets aux déformations osseuses et à l'expression de troubles locomoteurs (syndrome varus-valgus) que les poulets de souche à croissance lente. Un phénomène analogue n'est pas exclu chez les dindons de souche lourde mais son étude nécessiterait la mise au point d'une méthode de mesure fiable de la surface des canaux vasculaires. Toutefois, les problèmes locomoteurs rencontrés dans les 2 espèces sont difficilement comparables: les troubles s'expriment beaucoup plus tôt chez le poulet et se caractérisent par des déformations osseuses graves (Leterrier et Nys, 1992b) qui n'ont pas été retrouvées chez le dindon (Chérel et al, 1991a).

Notre étude confirme que la croissance osseuse diaphysaire est différente, à âge égal, tant sur le plan qualitatif que quantitatif chez les dindons des 2 souches étudiées. Elle est plus intense et plus précoce chez les dindons de souche lourde chez qui la forme de la diaphyse révèle l'action de contraintes mécaniques en relation probable avec le poids et la vitesse de croissance des animaux. La croissance diaphysaire est, en revanche, plus lente et plus régulière chez les dindons de souche fermière, chez qui les contraintes mécaniques liées au poids apparaissent moindres.

Les différences de poids importantes constatées entre les 2 lots d'animaux de notre étude rendent possible la comparaison des principaux paramètres de la croisance diaphysaire de dindons d'âges différents mais de poids sensiblement identiques; c'est le cas notamment des dindons de souche lourde et des dindons de souche fermière qui pèsent respectivement $600 \mathrm{~g}$ à 3 et $5 \mathrm{sem}, 1400 \mathrm{~g}$ à 5 et 8 sem et $2000 \mathrm{~g}$ à 6 et $10 \mathrm{sem}$.

A poids égal, tous les paramètres ne sont pas comparables dans les 2 souches : c'est ainsi que la surface diaphysaire totale (STd) n'est pas significativement différente aux 3 poids étudiés alors que la surface médullaire (SMd) est supérieure chez les dindons fermiers uniquement à $600 \mathrm{~g}$ et la surface corticale (SCd) est statistiquement supérieure chez les dindons lourds de $600 \mathrm{~g}$ et $1400 \mathrm{~g}$ (fig 9). À poids égal, le pourcentage de surface corticale (\% SCd) demeure toujours supérieur chez les animaux de souche lourde; la hauteur de la zone épaisse (ZMd) est supérieure chez les dindons lourds de $600 \mathrm{~g}$ et $1400 \mathrm{~g}$, et celle de la zone mine (ZMd) chez les animaux lourds de $2000 \mathrm{~g}$.

Des différences significatives existent donc entre les principaux paramètres de la croissance diaphysaire lorque l'on compare des animaux des 2 souches de poids identique. Ces différences ont tendance à diminuer, sans disparaître totalement, lorsque la comparaison intéresse le poids le plus élevé $(2000 \mathrm{~g})$. La plupart des paramètres sont, à poids comparable, supérieurs chez les dindons de souche lourde.

Ces résultats laissent supposer que, à poids identique, le squelette des dindons de souche lourde est soumis à des contraintes différentes de celles imposées aux dindons de souche fermière. Ces facteurs interviennent très tôt au cours de la vie des animaux et ont une influence sur les paramètres de surface et sur la forme de la corticale diaphysaire. Leur nature et leur rôle exact sur la croissance diaphy- 
saire et l'apparition éventuelle des troubles locomoteurs restent à évaluer.

\section{RÉFÉRENCES}

Abourachid A (1990) Étude morphofonctionnelle de l'appareil locomoteur de deux souches de dindons domestiques. Recherche d'une explication fonctionnelle aux boiteries des dindons ultra lourds. Thèse de doctorat d'université (université de Rennes I)

Chérel Y, Resch C, Wyers M (1991a) Aspects cliniques et fréquence des boiteries du dindon de chair. INRA Prod Anim 4, 311-319

Chérel $Y$, Beaumont $C$, Wyers $M$, Fleury $R$, Delavigne M (1991b) Estimations de la prévalence et de l'héritabilité de la dyschondroplasie tibiale du dindon. Avian Pathol 20, 387401

Chabeauti E (1988) Le squelette du dindon en croissance : étude de la vitesse d'apposition osseuse de la diaphyse tibiale par une méthode de double marquage à l'oxytétracycline. Thèse pour le diplôme d'État de docteur vétérinaire (Nantes)

Dammrich K, Rodenhoff G (1970) Skelettveönderungen bei Masküken. Zentralbl Veterinaermed Reihe B 17, 131-146

Frost HM (1973) Bone remodeling and its relationship to metabolic bone diseases. Orthopaedic lectures, vol III (CC Thomas, ed) Springfield, USA

Hutzchnenreuter $P$, Brummer $C$ (1984) Embedding, cutting and grinding methods to produce undecalcified cortical bone sections over the whole bone. Acta Anat 118, 201-204

Lanyon LE (1984) Functional strain as a determinant for bone remodeling. Calcif Tissue 36 , 56-61

Leblanc B, Wyers $M$, Cohn-Bendit F, Legall JM, Thibault E, Florent JM (1986) Histology and histomorphometry of the tibia growth in two turkey strains. Poultry Sci 65, 1787-1795

Leterrier C, Nys Y (1992a) Composition, corticale structure and mechanical properties of chicken tibiotarsi: effect of growth rate. $\mathrm{Br}$ Poult Sci 33, 925-939

Leterrier C, Nys Y (1992b) Clinical and anatomical differences in varus and valgus deformi- ties of chick limbs suggest different aetiopathogenesis. Avian Pathol 21, 431-444

Mariau V (1988) Le squelette du dindon en croissance : étude histologique, qualitative et quantitative du développement de la diaphyse tibiale. Thèse pour le diplome d'état de docteur vétérinaire (Nantes)

Pellegrino ED, Biltz RM (1983) A chick bone model for evaluating radial bone growth. 1 . Effects of Vitamin D3 deficiency. Poult Sci 62, 2083-2087

Pratt CWM (1961) The effect of age on the arrangement of fibres in the bone matrix of the femur of the domestic fowl. J Anat 95,110-122

Ranaweera KNP, Wise DR (1981) The effect of trienbolone acetate on carcass composition, conformation and skeletal growth of turkeys. Br Poult Sci 22, 105-114

De Ricqles A (1975) Recherches paléohistologiques sur les os longs des Tétrapodes. VII. Sur la classification, la signification fonctionnelle et l'histoire des tissus osseux des Tétrapodes (1re Partie). Ann Paléontol Vertebr 61, 51-129

De Ricqles A (1977) Recherches paléohistologiques sur les os longs des Tétrapodes. Vil. Sur la classification, la signification fonctionnelle et l'histoire des tissus osseux des Tétrapodes. 2e partie (fin). Ann Paleontol Verteb $63,133-160$

Riddell C (1975a) Pathology of developmental and metabolic disorder of the skeleton of domestic chickens and turkeys. I. Abnormalities of genetic or unknown aetiology. Vet Bull 45, 629-640

Riddell C (1975b) Pathology of developmental and metabolic disorder of the skeleton of domestic chickens and turkeys. II. Abnormalities due to nutritional or toxic factors. Vet Bull 45, 705-718

Riddell C (1981) Skeletal Deformities in Poultry. Adv Vet Sci Comp Med 25, 277-310

Rodenhoff G, Dammrich K (1971) Über die Beeinfflussung der Skelettis der Mästhahnchen durch Haltung und Auslauf im Freien. Zentralbl Veterinaermed Reihe A 18, 297309

Sandhu HS, Jande SS (1981) Radioisotopic and morphometric evaluation of the effects of $\beta$ aminopropionitrile on chick bone matrix formation and its mineralization. Acta Anat 111, 281-288 
Sanger VL, Dahlgren RR, Cover MS, Langham RF (1974) Skeletal disease and locomotor problems in turkeys. Avian Dis 18, 378-393

Schenck RK, Olah AJ, Herrmann W (1984) Preparation of calcified tissues for light microscopy. In: Methods of calcified tissue preparation (Dickson GR, ed). Elsevier Science, Amsterdam, 1-56

Villanueva AR, Mehe LA (1977) Modifications of the Goldner and Gomori one-step trichrome stains for plastic embedded thin sections. Am J Med Techn 43, 536-538

Wise DR (1975) Skeletal abnormalities in table poultry. A Review. Avian Pathol 4, 1-10

Wyers $M$, Hamon JC, Chérel $Y$, Plassiart G (1993) Histology and histomorphometry of the tibial diaphysal growth in two turkeys strains during the first six weeks after hatching. Anat, Histol, Embryol 22, 48-58 